Paper

\title{
Quantitative and qualitative discrimination of task periods from non-task periods by recurrence plots
}

\author{
Masako Sugai ${ }^{1 a)}$ and Masaharu Adachi ${ }^{16)}$ \\ ${ }^{1}$ Graduate School of Advanced Science and Technology, Tokyo Denki University, \\ 5 Senjyu-Asahi-cho, Adachi-ku, Tokyo 120-8551, Japan \\ a) 12ude01@ms.dendai.ac.jp \\ b)adachi@eee.dendai.ac.jp
}

Received October 22, 2015; Revised December 12, 2015; Published April 1, 2016

\begin{abstract}
Recurrence plots are useful tools for visualizing the inner structure of a time series, and they have been applied to physiological data to examine brain activity. However, these studies have focused on qualitative analysis of the data. In this study, we used unthresholded recurrence plots of near-infrared spectroscopy (NIRS) data to detect the difference in brain activity between task periods and non-task periods. Histograms derived from the recurrence plots showed a statistical difference between the periods. Throughout the pre-task period, task period, and post-task period, the histogram kept its shape statistically and shifted horizontally according to the period. Therefore, the changes in dynamical systems describing brain activity were observed as changes in histograms derived from NIRS data.
\end{abstract}

Key Words: NIRS (near infra-red spectroscopy), recurrence plots, histograms, Friedman test

\section{Introduction}

Near infra-red spectroscopy (NIRS) is a low-power, non-invasive method for measuring brain activity $[1,2]$. NIRS instruments are small compared with other instruments, such as those for functional magnetic resonance imaging (fMRI) or magnetoencephalography (MEG). Recently, portable NIRS instruments have become available, and so subjects can include inpatients or outpatients and newborn infants in addition to healthy people.

Despite these advantages, the NIRS data contain two unknown constants: the baseline $(B)$ and the optical path length $(O P L)$. Moreover, NIRS data are often contaminated with various kinds of noise such as scalp blood flow, cardiac circulation, or motion artifacts. In this study, the hidden structure in noisy NIRS data is visualized with recurrence plots. Furthermore, a statistical difference between the task period and non-task period in the NIRS data is extracted with histograms derived from the recurrence plots.

This is the first study where the dynamical changes linked to brain activity have been observed by statistically significant changes in histograms derived from NIRS data. 


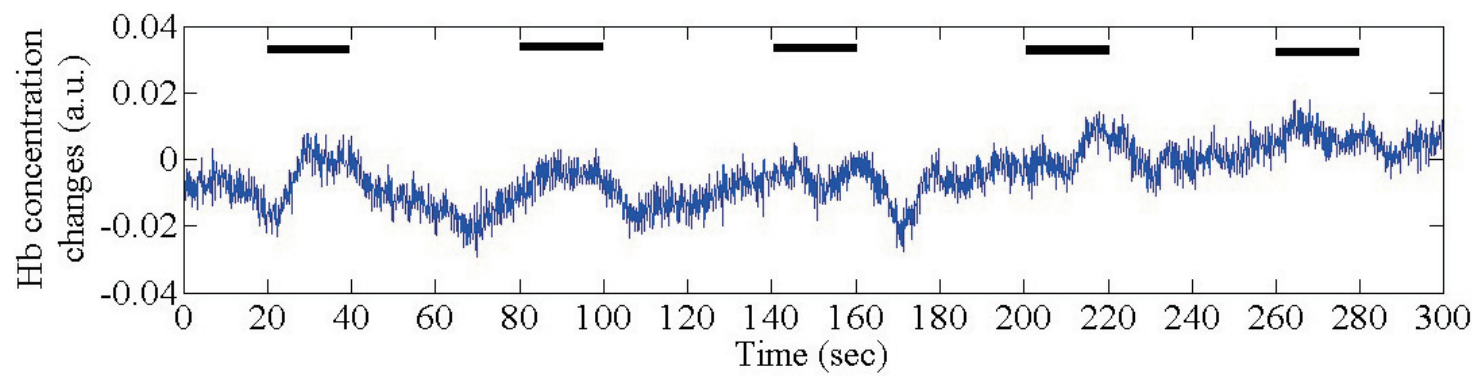

Fig. 1. Example of NIRS data. Black bars in the field indicate the task periods.

\subsection{Neurovascular coupling}

The relationship between neuronal activity and blood flow is called neurovascular coupling [3]. When neurons in the brain are activated, they require more oxygen. The volume of blood flow increases over the cortical surface to supply the area with oxygen. Oxygen is transported through the cerebral blood vessels, carried by the metalloprotein hemoglobin. Thus, changes in hemoglobin concentration occur in regions where blood flow increases.

\subsection{Formulation of NIRS time series}

NIRS data contain the unknown constants $B$ and $O P L$. Let $x(t)$ be a time series of the changes in oxygenated hemoglobin concentration at the measuring point, and $y(t)$ be a time series obtained by NIRS. Then $y(t)$ can be written as

$$
y(t)=B+O P L \cdot x(t)+n(t)
$$

where $B$ is the baseline in the initial state $(t=0)$ and $n(t)$ is the noise term.

There are two major problems with the analysis of NIRS data. First, because the brain is active even in a steady state, $B$ is a constant and depends on the initial condition $(t=0)$. Second, $O P L$ differs between measuring points, near-infrared wavelengths, and subjects. NIRS data contain physiological noise (cardiac circulation, respiration [2], and scalp blood flow [4,5]), motion artifacts [6], and mechanical noise. An example of NIRS data is shown in Fig. 1. It is difficult to identify the task period in the figure visually.

\subsection{Previous studies}

NIRS data analysis has been performed with methods such as subtraction [7], a general linear model (GLM) [8,9], and Fourier transforms (FT) [10].

NIRS data has been analyzed by subtracting task period data from the non-task period data. This method is simple and can compensate for the effect of $B$. However, it still suffers from the problems with $O P L$, and the data from different measuring points cannot be compared directly.

The hemodynamic reactive function (HRF) and GLM have been used for NIRS data analysis. These methods are based on fMRI analysis. However, the assumption that the error terms follow a normal distribution is so strict that the method cannot be used for tasks that contain motion artifacts; even static tasks may not be suitable due to the error terms.

FT has been used to analyze NIRS data with a method similar to that used in electroencephalography or MEG. However, owing to the low temporal resolution in NIRS measurement and the small number of data points, this method is not standard in NIRS data analysis.

In this study, reconstructed attractors were obtained from the data. After signal averaging, we drew the recurrence plots with Euclidean distance to compensate for the effect of $B$. Moreover, to remove the effect of individual differences in $O P L$ and neuronal activation, we normalized the elements of the recurrence plots by the maximum values in all the elements of each subject's recurrence plots. To compare the task period and non-task period, histograms were constructed from the recurrence plots. To avoid using model-based analysis, a non-parametric multi-comparison test (Friedman test) was applied to the histograms. In recurrence plots, the periodic sequential changes and stochastic changes 
appear as regular patterns and random patterns, respectively. Therefore, the gradation of recurrence plots corresponds to spectral decomposition. If the number of data points in the time series is $N$, the number of elements in the histograms derived from recurrence plots is $O\left(N^{2}\right)$. Hence, more data points can be used from histograms derived from the recurrence plots.

\section{Embedding into high-dimensional phase space}

Time series do not always show their characteristics in one-dimensional space because of their parameters and noise [11]. To extract the hidden structure of the given time series, the obtained data are embedded into high-dimensional phase space. The embedding parameters time delay $\tau$ and embedding dimension $d$ are required. With these parameters, the reconstructed attractors $Y(t)(t=1,2, \ldots)$ are represented as

$$
Y(t)=(y(t), y(t+\tau), y(t+2 \tau), \ldots, y(t+(d-1) \tau)) .
$$

\subsection{Time delay}

To determine $\tau$, we choose the minimum positive integer $\tau$ that gives the minimal average mutual information of a given time series.

Prior to calculating time delays, we address the average mutual information. Mutual information is one of the nonlinear time series analysis, which enables us to quantify the strength of connection between two time series. In detail, let $X=\{x(t)\}_{t}$ and $Y=\{y(t)\}_{t}$ be two sets of random variables. Then mutual information $I(X, Y)$ is defined as

$$
\begin{aligned}
I(X, Y) & =H(X)-H(X \mid Y) \\
& =-\sum_{t} p(x(t), y(t)) \log _{2} \frac{p(x(t)) p(y(t))}{p(x(t), y(t))}
\end{aligned}
$$

where,

$H(X)$ : Shannon's entropy of X,

$H(X \mid Y)$ : conditional entropy of $\mathrm{X}$ conditioned on $\mathrm{Y}$,

$p\left(x_{i}\right)$ : kernel density estimation of $x_{i}$,

$p\left(x_{i}, y_{j}\right)$ : joint probability distribution of $X=x_{i}, Y=y_{j}$.

Here we estimate the probability function as data-driven one. The methods for defining the number of bins are reported in [12-14]. Our goal is to discriminate the difference between the periods in a quantitative way, so within the range of these previous reports [12-14], we define the number of bins as 40 .

In Eq. (4), let $y(t)$ be replaced with $x(t+\tau)$ and calculate the average of $I(X, Y)$ over $t$. Then time delay $\tau$ is defined as the smallest integer that gives the minimal average mutual information.

\subsection{Embedding dimension}

Proceeding to time delay, we calculated an embedding dimension $d$ with the false nearest neighbor approach $(F N N)$. The method of $F N N$ is as follows.

Let $\left(\{Y(t)\}_{t}\right)$ be reconstructed vectors after embedding into $m$ dimensional space with time delay $\tau$, i.e.,

$$
Y(t)=(y(t), y(t+\tau), y(t+2 \tau), \ldots, y(t+(m-1) \tau)) .
$$

For ${ }^{\forall} k,{ }^{\exists} n(k)$ s.th. $Y(n(k))$ is the nearest neighbor of $Y(k)$. Then, $D_{m}(Y(k), Y(n(k)))$, Euclidean distance between $Y(k)$ and $Y(n(k))$ in $m$ dimensional space, satisfies

$$
D_{m}(Y(k), Y(n(k)))^{2}=\sum_{i=1}^{m}\{y(k+(i-1) \tau)-y(n(k)+(i-1) \tau)\}^{2} .
$$

Similarly, in $m+1$ dimensional space, Euclidean distance between $Y(k)$ and $Y(n(k))$ satisfies 


$$
D_{m+1}(Y(k), Y(n(k)))^{2}=\sum_{i=1}^{m+1}\{y(k+(i-1) \tau)-y(n(k)+(i-1) \tau)\}^{2} .
$$

Suppose the dimension changes from $m$ to $m+1$, let $R_{L}$ based on $m$ and $Y(k)$ be defined as

$$
\begin{aligned}
R_{L} & =\sqrt{\frac{D_{m+1}(Y(k), Y(n(k)))^{2}-D_{m}(Y(k), Y(n(k)))^{2}}{D_{m}(Y(k), Y(n(k)))^{2}}}, \\
& =\frac{|y(k+m \tau)-y(n(k)+m \tau)|}{D_{m}(Y(k), Y(n(k)))} .
\end{aligned}
$$

If $R_{L}$ is larger than threshold $z_{0}$, then $Y(n(k))$ is called as the false nearest neighbor of $Y(k)$.

The embedding dimension $d$ is defined as the smallest integer $m$ which satisfy that, if $m$ changes to $m+1$, then $R_{L} \leq Z_{0}$ for ${ }^{\forall} k[16]$.

According to Takens approach $[17,18]$, the methods mentioned above provide the embedding of the original time series. Based on the previous reports [19-22], in this study, we put $z_{0}$ as 15 for the analysis.

\subsection{Recurrence plots}

Recurrence plots are a useful tool for visualizing time series [23-27]. Recurrence plots are strictly defined as a binary matrix. In a broader definition, the term "recurrence plots" refers to the distance matrix among reconstructed attractors $[28,29]$. In physiological studies, distance matrices are sometimes treated as recurrence plots [30]. Therefore, for clinical applications or brain computer interfaces (BCI), distance matrices are used as recurrence plots. For example, let $x(t)$ be given time series and $Y(t)$ be reconstructed attractors of $x(t)$. The recurrence plots $R_{Y}=\left\{R_{i j}\right\}(i, j=1,2, \ldots)$ of $Y(t)$ are defined as

$$
R_{i j}=D_{d}(Y(i), Y(j)),(i, j=1,2, \ldots),
$$

where $D_{d}(\mathrm{~A}, \mathrm{~B})$ expresses the Euclidean distance between A and B in the $d$-dimensional reconstructed phase space.

We acquired signal-averaged data from raw NIRS data, and then divided it into the pre-task period, task period, and post-task period. We obtained five kinds of time series from each subject. Then, we embedded the time series into high-dimensional phase space and calculated the recurrence plots.

A representative recurrence plot in the embedded space of raw NIRS data is shown in Fig. 2. The texture of the recurrence plots has a periodic pattern. The distance between the task periods is small, whereas the distance between the task and non-task periods is large.

\subsection{Histograms}

Recurrence plots are used to extract the characteristic structure of the NIRS data. The texture and contrast of recurrence plots provide information about the hidden structure of the time series. As described in this section, histograms based on recurrence plots were used to differentiate statistically among the task and non-task periods.

First, signal averaging was performed to improve the signal-to-noise ratio. Recurrence plots of the signal-averaged data were calculated. The signal-averaged data consists of the pre-task period, task period, and post-task period. The embedding parameters of the signal-averaged data were the same as those of each period, allowing the three periods in the recurrence plot, called sub-recurrence plots, to be extracted from the signal-averaged data and compared directly with each other.

To exclude the effect of the diagonal line, the elements of the upper triangle in the sub-recurrence plots were examined (Fig. 3). For constructing the histograms based on the recurrence plots, we set the number of bins as 40 according to the previous reports [12-14]. 


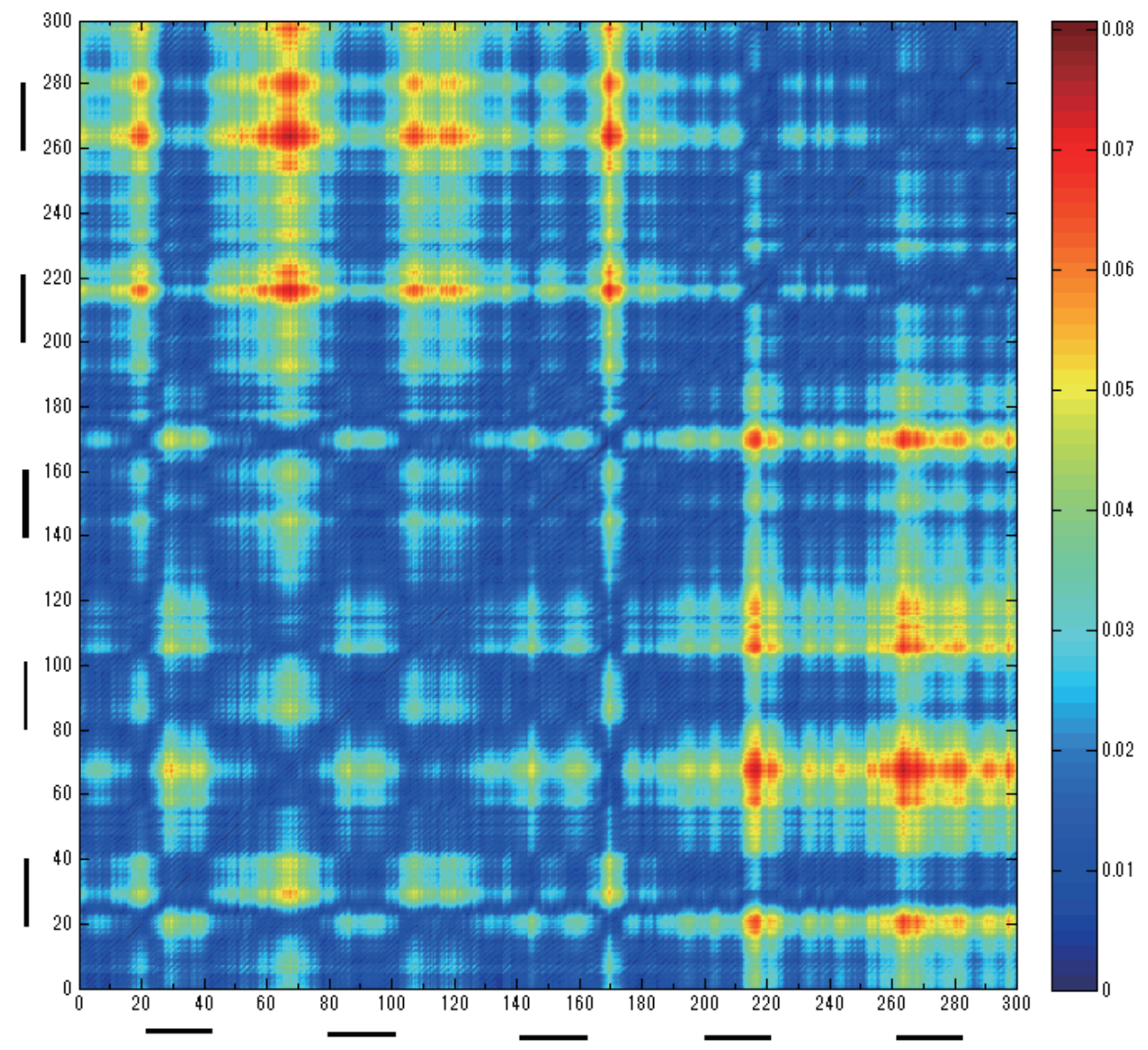

Fig. 2. A representative recurrence plot of raw NIRS data in reconstructed space (subject 4). Black bars along the axes indicate the five task periods, each of which is $20 \mathrm{~s}$ in duration $(\tau=4, d=5)$.

(a)

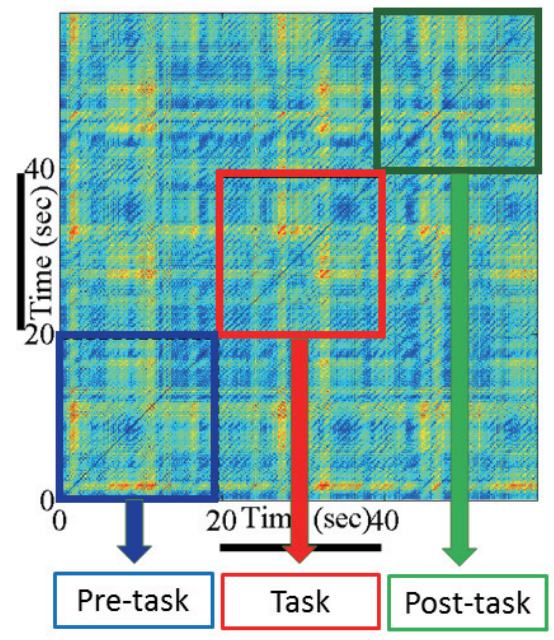

(b)

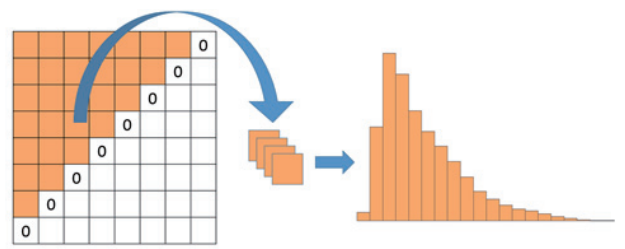

(c)

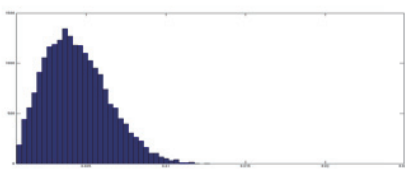

\section{Task}

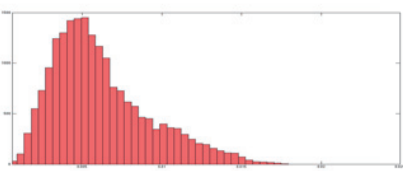

Post-task

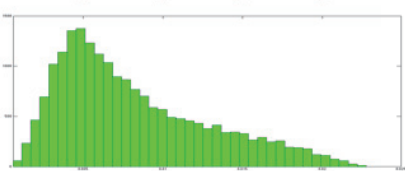

Fig. 3. Schematic of procedure for pre-task, task and post task periods. (a) Three sub-recurrence plots are taken out of the normalized recurrence plots of signal-averaged data. (b) The upper triangular elements in each recurrence plot were used to construct the histogram. (c) Three histograms were obtained from each subject's recurrence plots. 


\section{Experimental design}

\subsection{Subjects and methods}

Twenty-two healthy right-handed subjects participated in the experiment. They were asked to grasp their right hands during the task periods according to the blinking signals displayed on a computer monitor. A block of three periods, namely, the pre-task period (20 s), task period (20 s), and post-task period $(20 \mathrm{~s})$, was repeated five times for each measurement. In the task period, the signals flashed red once a second, and the monitor displayed black otherwise. In right-handed subjects, the left primary motor cortex (M1) is activated during right-grasping [31], thus the NIRS measuring point was on the left M1, corresponding to $\mathrm{C} 3$ in the international 10-20 system [32]. NIRS data were obtained with an NIRS instrument (OMM-3000, Shimadzu Corp. Kyoto, Japan; sampling interval: $130 \mathrm{~ms}$ ) and the data processing was performed with MATLAB 2013 (The MathWorks, Inc., Natick, MA).

All the measurements were done in a quiet, lit room. Written informed consent was obtained from all subjects before the experiments were conducted. All procedures were done in accordance with the tenets of the Declaration of Helsinki and under the approval of the Ethics Committee of Tokyo Denki University.

\subsection{NIRS data analysis}

To decrease the signal-to-noise ratio, we performed signal averaging on the time series data, and embedded the signal-averaged data into high-dimensional phase space. Recurrence plots were applied to the reconstructed attractors based on the signal-averaged data to visualize the difference between the task period and non-task period. Furthermore, to detect the statistical difference between the pre-task period and the task period, the histograms derived from the elements of recurrence plots were examined. The three sub-recurrence plots were extracted from the recurrence plots derived from the signal-averaged data. For comparison, we used the same horizontal line range and same number of bins for the three histograms.

To detect the statistical difference in histograms among the periods, we performed a non-parametric multi-comparison statistical test (Friedman test [33]) for six statistics (average, median, mode (the order of the bin that gave the maximum), maximum, skewness, and kurtosis). Post hoc Scheffe tests [34] were performed. The level of significance, $\alpha$, was defined as $5 \%$.

\section{Results}

\subsection{Embedding parameters}

Five pairs of embedding parameters were calculated for raw NIRS data, signal-averaged data, and the data for the three periods. The results of the embedding parameters in 22 subjects are shown in Fig. 4.

Friedman tests were used to perform the distribution-free test. After obtaining five pairs of embedding parameters from each subject, we detected the significant differences among each embedding parameter $(\alpha=0.05)$. Furthermore, post hoc Scheffe tests were performed to detect significant differences between two of five embedding parameters. There was no significant difference among time delays $(\mathrm{Q}=6.94, p=0.139)$, but significant differences were found among embedding dimensions $(\mathrm{Q}=56.2, p<0.001)$.

There were significant differences between the raw NIRS data and the other four data sets. Based on the Scheffe test, there was no significant difference among these four data sets. The statistical results are shown in Table I.

\subsection{Recurrence plots}

After calculating the embedding parameters, we embedded the NIRS data into high-dimensional space. To obtain the recurrence plots in each period, we constructed the recurrence plots from the signal-averaged data. Three groups of recurrence plots were taken from the signal-averaged plot. Two examples of recurrence plots for the signal-averaged data in representative subjects are shown in Fig. 5, in which the difference in textures among the periods is visible. The recurrence plot of subject 
(a) Raw data

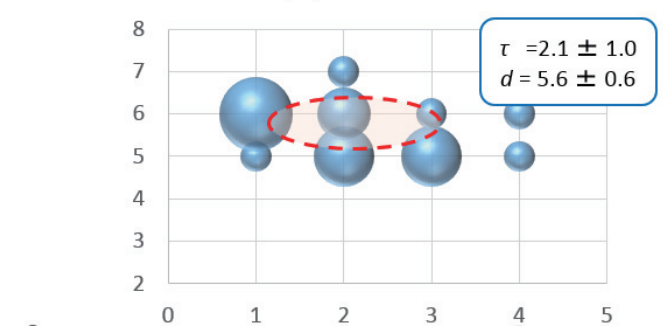

(b) Signal-averaged

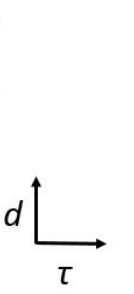

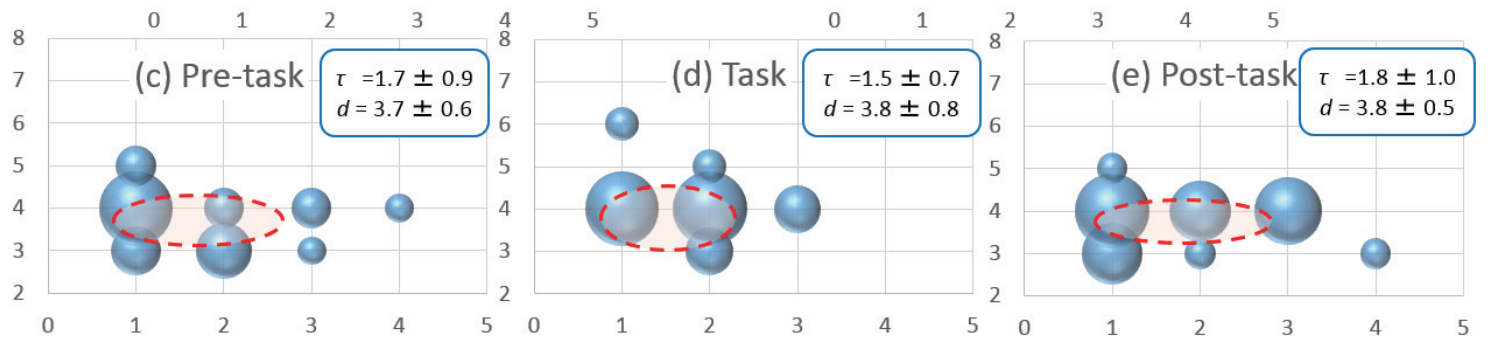

Fig. 4. Bubble charts and the five pairs of embedding parameters for the (a) raw data, (b) signal-averaged data, (c) pre-task period, (d) task period, and (e) post-task period. The radius of the bubbles reflects the number of subjects. Red dotted ellipses show the range of average \pm standard deviation in the embedding parameters. The range of all axes is the same to allow direct comparison.

Table I. Results of the multiple comparison (Scheffe test) in the embedding dimension. The elements in the lower triangle are statistical values among the periods, and the elements in the upper triangle are the corresponding $p$-values. Sg-Avg: signal-averaged data.

\begin{tabular}{|l|c|c|c|c|c|}
\hline & Raw data & Sg-Avg & Pre-task & Task & Post-task \\
\hline Raw data & $*$ & $<0.005$ & $<0.001$ & $<0.001$ & $<0.001$ \\
\hline Sg-Avg & 16.31 & $*$ & 0.34 & 0.34 & 0.45 \\
\hline Pre-task & 38.01 & 4.52 & $*$ & 1 & 0.99 \\
\hline Task & 38.01 & 4.52 & 0 & $*$ & 0.99 \\
\hline Post-task & 35.43 & 3.66 & 0.05 & 0.05 & $*$ \\
\hline
\end{tabular}
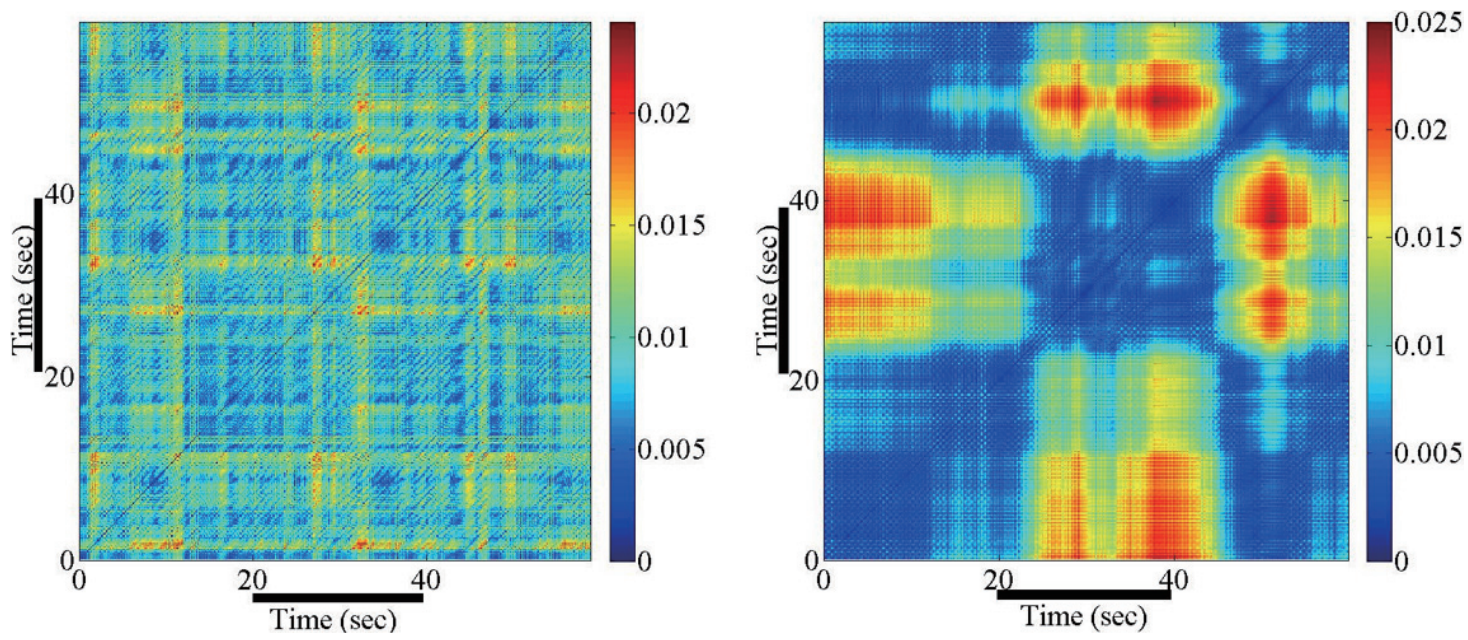

Fig. 5. Recurrence plots for the signal-averaged data in two representative subjects. Left: subject $3(\tau=2, d=4)$, right: subject $4(\tau=3, d=4)$. Both recurrence plots are based on the signal-averaged data. The black bars indicate the task periods.

3 , is smooth although the texture differs between the pre-task period and task period. In contrast, in subject 4 , the contrast in the recurrence plots is clear. Moreover, compared with Fig. 2, it is easier to detect the task period in the recurrence plots. 
(a) Average

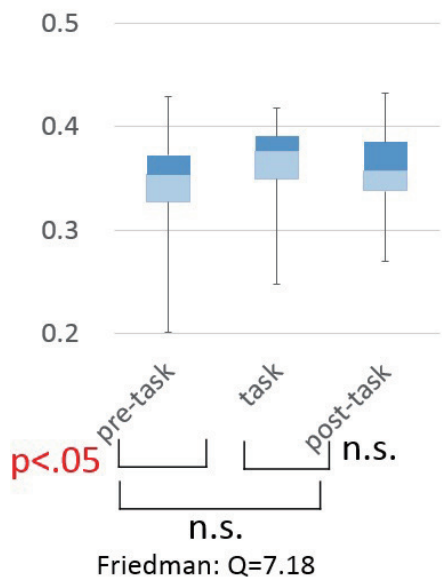

(b) Median

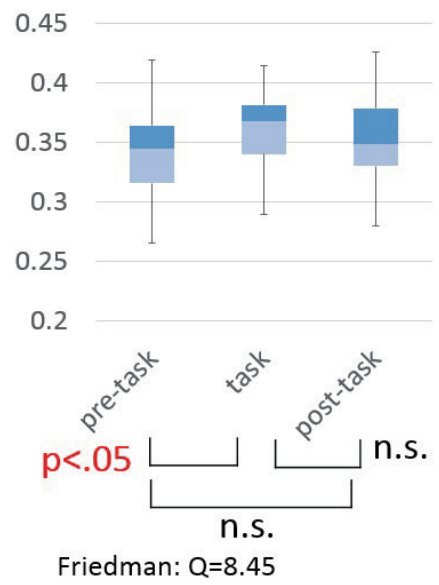

(c) Mode

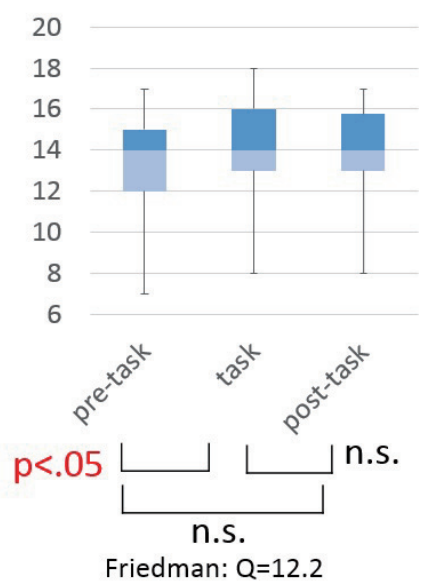

Fig. 6. Results of the interquartile and statistical test for the (a) average, (b) median, and (c) mode. n.s.: not significant.

(a) Maximum

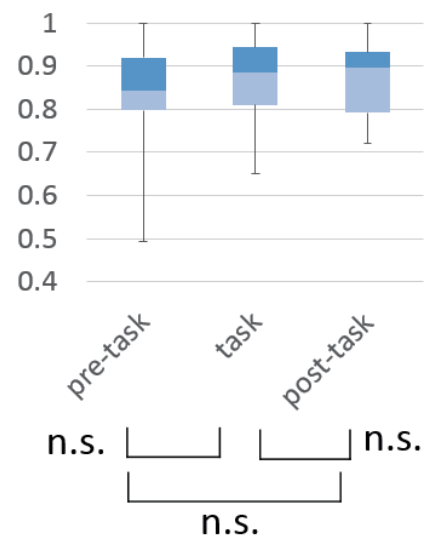

Friedman: $\mathrm{Q}=0.81$ (b) Skewness

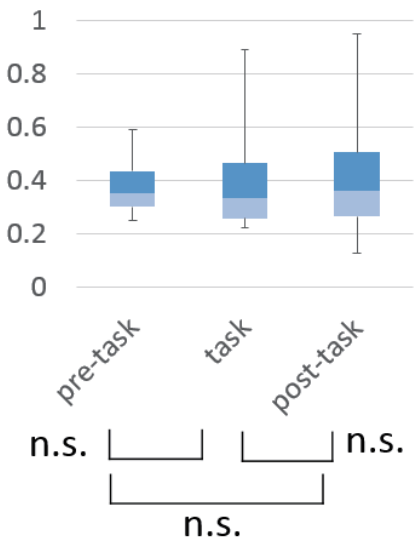

Friedman: $\mathrm{Q}=0.81$ (c) Kurtosis

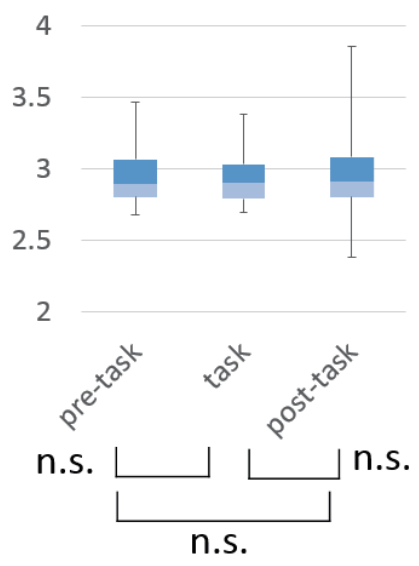

Friedman: $\mathrm{Q}=0.27$

Fig. 7. Results of the interquartile and statistical test for the (a) maximum, (b) skewness, and (c) kurtosis. n.s.: not significant.

\subsection{Histograms}

Prior to the statistical analysis of the three periods, we checked the maximum elements of the recurrence plots based on the signal-averaged data. The maximum varied widely from 0.024 to 0.258 among subjects. Therefore, we normalized the recurrence plots by the maximum element. The three parts of the recurrence plots were extracted from the normalized recurrence plot.

Friedman tests were performed to determine whether there was a significant difference among the periods (Figs. 6 and 7). For multiple comparisons, Scheffe tests were used to detect significant differences between periods with a significance level of $\alpha=0.05$. The results are shown in Tables II-IV.

For the average, median, and mode, Friedman test showed that there were significant differences among the three periods (mean: $\mathrm{Q}=7.18, p<0.05$; median: $\mathrm{Q}=8.45, p<0.05$; mode: $\mathrm{Q}=12.2$, $p<0.05$ ). The results are shown in Fig. 6 . The Friedman test showed that there were significant differences among the periods. The Scheffe test showed that there were significant differences between the pre-task period and task period (mean: $\mathrm{Q}=6.57, p<0.05$; median: $\mathrm{Q}=7.36, p<0.05$; mode: $\mathrm{Q}=6.24, p<0.05$ ). However, for three of the statistics, there were no significant differences between the post-task period and the other two periods. The results are shown in Tables II-IV.

In contrast, for the maximum, skewness, and kurtosis, there were no significant differences among the three periods (maximum: $\mathrm{Q}=0.81, \mathrm{p}=0.67$; skewness: $\mathrm{Q}=0.81, \mathrm{p}=0.67$; kurtosis: $\mathrm{Q}=0.27$, $\mathrm{p}=0.87)$. The results are shown in Fig. 7. In MATLAB 2013, kurtosis of the normal distribution 
Table II. Results of the multiple comparison (Scheffe test) of the histogram mean among the periods. The elements in the lower triangle are the test statistics, and the elements in the upper triangle are the $p$-values. The median of mean was significantly larger in task period than in the pre-task period $(\mathrm{Q}=6.57, p<0.05)$. However, there were no significant differences between the post-task period and the other two periods.

\begin{tabular}{|l|c|c|c|}
\hline & Pre-task & Task & Post-task \\
\hline Pre-task & $*$ & $<0.05$ & 0.15 \\
\hline Task & 6.57 & $*$ & 0.83 \\
\hline Post-task & 3.84 & 0.36 & $*$ \\
\hline
\end{tabular}

Table III. Results of multiple comparison (Scheffe test) of the histogram median among the periods. The elements in the lower triangle are the test statistics, and the elements in the upper triangle are the $p$-values. Scheffe test showed that the median of median was significantly larger in the task period than in the pre-task period $(\mathrm{Q}=7.36, p<0.05)$. However, there were no significant differences between post-task period and the rest of the two periods.

\begin{tabular}{|l|c|c|c|}
\hline & Pre-task & Task & Post-task \\
\hline Pre-task & $*$ & $<0.05$ & 0.07 \\
\hline Task & 7.36 & $*$ & 0.90 \\
\hline Post-task & 5.11 & 0.20 & $*$ \\
\hline
\end{tabular}

Table IV. Results of multiple comparison (Scheffe test) of the histogram mode among the periods. The elements in the lower triangle are the test statistics, and the elements in the upper triangle are the $p$-values. The median of mode was significantly larger in the task period than in the pre-task period $(\mathrm{Q}=6.24, p<0.05)$. However, there were no significant differences between the post-task period and the other two periods.

\begin{tabular}{|l|c|c|c|}
\hline & Pre-task & Task & Post-task \\
\hline Pre-task & $*$ & $<0.05$ & 0.29 \\
\hline Task & 6.24 & $*$ & 0.64 \\
\hline Post-task & 2.44 & 0.88 & $*$ \\
\hline
\end{tabular}

Table V. Results for the Wilcoxon signed rank test for skewness.

\begin{tabular}{|c|c|c|c|}
\hline & Pre-task & Task & Post-task \\
\hline$Z$-score, $p$-value & $Z=4.09, p<0.001$ & $Z=4.09, p<0.001$ & $Z=4.09, p<0.001$ \\
\hline
\end{tabular}

Table VI. Results for the Wilcoxon signed rank test for kurtosis.

\begin{tabular}{|c|c|c|c|}
\hline & Pre-task & Task & Post-task \\
\hline$Z$-score, $p$-value & $Z=-1.12, p=0.263$ & $Z=-1.70, p=0.090$ & $Z=-1.15, p=0.249$ \\
\hline
\end{tabular}

is defined as three. To examine the histograms, Wilcoxon signed rank tests were performed to assess skewness and kurtosis. The results for skewness are shown in Table V. The skewnesses have a positive median. The null hypothesis was that the median skewness was equal to zero. However, in all periods, the null hypothesis was rejected, so skewness followed a distribution biased in the negative direction. The results for kurtosis are shown in Table VI. The null hypothesis was that the median kurtosis was equal to 3 (i.e., that the distributions are univariate normal distributions). In every period, the test failed to reject this null hypothesis, at a significance level of $5 \%$.

\section{Discussion}

The embedding of NIRS data into high-dimensional space has been reported [35, 36]. However, there is no study that has examined the statistical difference between the task period and non-task period. This study is the first to investigate the dynamical changes that describe the brain activity with the statistical changes in histograms derived from NIRS data in one dimension.

With our proposed method, the effects of $B$ and $O P L$ can be canceled. $B$ can be subtracted when 
the recurrence plots are calculated. Additionally, normalizing recurrence plots compensates for the effect of $O P L$ and the individual differences in brain activation, enabling us to compare the data among subjects.

Among the physiological noises, scalp blood flow can be treated as a constant because of its shallow pathway [37], which is canceled in recurrence plots with subtraction. In general, periodic noises appear as the regular patterns in recurrence plots. However, some physiological noises such as heart beats consist of different frequency spectrum [38]. Therefore, regarding the physiological noises including heart beats and so on, we will discuss the matter in further study.

The embedding parameters remain the same throughout the three periods for each subject. We analyzed raw NIRS data, signal-averaged data, and pre-task period, task period, and post-task period data. If the embedding parameters differ statistically among the periods, each segment must be analyzed with its own embedding parameters. However, there were no significant differences in the embedding parameters between signal-averaged data, the pre-task period, the task period, and the post-task period. Therefore, the three periods could be compared as sub-recurrence plots within the recurrence plots of a signal-averaged time series.

Recurrence plots can visualize the difference between periods. For example, in Fig. 5, in subject 3, the whole texture of the recurrence plots appears smooth. However, the textures of two of the three periods are different, suggesting that they have different hidden structures. In contrast, in subject 4, there were marked differences in the recurrence plots, which indicated differences in amplitude between the periods.

Recurrence plots can also be used to discriminate between periods statistically. In our study, the Friedman test was used as a non-parametric multi-comparison test. The null hypothesis was that there was no significant difference in statistics between periods. The results of the Friedman test for histograms (Fig. 6), showed that for the mean, median, and mode, the null hypothesis was rejected. Moreover, with Scheffe test, there were significant differences between the pre-task period and the task period for the average, median, and mode. Hence, the recurrence plots discriminated between the pre-task period and task period visually and statistically.

The Friedman test showed that for the maximum, skewness, and kurtosis there was no significant difference among the periods (Fig. 7). According to the Wilcoxon signed rank test, the skewness followed the distribution for which the median was larger than zero (Table V). Thus, the rough sketch of the histograms was left-biased throughout the three periods. The Wilcoxon test also showed that the distribution of kurtosis is not significantly different from what would be obtained for univariate normal histograms (Table VI). Therefore, throughout the periods, the histograms derived from the recurrence plots kept its shape statistically and shifted vertically in the positive direction from the pre-task to the task period, and then, from the task period to post-task period, it shifted in the negative direction back to the steady state. In other words, the dynamical changes that describe the brain activity emerged as quantitative changes in the histograms derived from the NIRS data.

These results are consistent with the hemodynamic response occurring gradually after the neuronal activity and returning to the steady state slowly over a maximum of $10 \mathrm{~s}$ after neuronal activation stops. The intentional control of a motor sequence requires a wired neuronal activation network [39, 40], and the histogram shifts in the positive direction during the task period because of its activation. Our proposed method shows that statistically significant differences in histograms derived from NIRS data describe the dynamical changes in brain activity.

Recently, the measurements with NIRS are getting more familiar in laboratory, in clinical scene and BCIs. Our proposed method is appropriate for real-time analysis, therefore, it contributes to many aspects of NIRS studies.

\section{Conclusion}

To determine the differences among task and non-task periods in NIRS data, recurrence plots were used. The histograms derived from recurrence plots for the pre-task period, task period, and post-task period revealed the statistical difference between the pre-task period and task period. Furthermore, throughout the periods, the histograms maintained their shape statistically and shifted vertically in 
accordance with the periods.

Therefore, recurrence plots can distinguish periods visually and statistically, and thus provide information about dynamical changes in the brain.

\section{Acknowledgments}

The authors would like to thank Prof. Yoshihiko Horio (Tokyo Denki University) for his helpful advice. The authors would like to thank anonymous reviewers for their fruitful suggestions and comments.

This work was partially supported by JSPS KAKENHI Grant Number (C) 25420835.

\section{References}

[1] A. Torricelli, D. Contini, A. Pifferi, M. Caffini, R. Re, L. Zucchelli, and L. Spinelli, "Time domain functional NIRS imaging for human brain mapping," NeuroImage, vol. 85, no.1, pp. 2850, January 2014.

[2] A. Roe and W. Anna, Imaging the brain with optical methods, Springer, New York, 2010.

[3] M.A. Franceschini and D.A. Boas, "Noninvasive measurement of neuronal activity with nearinfrared optical imaging," NeuroImage, vol. 21, no. 1, pp. 37-386, January 2004.

[4] T. Takahashi, Y. Takikawa, R. Kawagoe, S. Shibuya, T. Iwano, and S. Kitazawa, "Influence of skin blood flow on near-infrared spectroscopy signals measured on the forehead during a verbal fluency task," NeuroImage, vol. 57, no. 3, pp. 991-1002, August 2011.

[5] M. Sugai and M. Adachi, "Identification of scalp blood flow in NIRS data based on Granger causality," Proc. BIBE'13, Greece, November 2013.

[6] S. Brigadoi, "Motion artifacts in functional near-infrared spectroscopy: A comparison of motion correction techniques applied to real cognitive data," NeuroImage, vol. 85, pp. 181-91, 2014.

[7] T. Hashimoto, Y. Minagawa-Kawaia, and S. Kojima, "Motion illusion activates the visual motion area of the brain: A near-infrared spectroscopy (NIRS) study," Brain Research, vol. 1077, no. 1, pp. 116-122, 2006.

[8] M. Plichta, S. Heinzel, A. Ehlis, P. Pauli, and A. Fallgatter, "Model-based analysis of rapid event-related functional near-infrared spectroscopy (NIRS) data: A parametric validation study," NeuroImage, vol. 35, pp. 625-634, 2007.

[9] A. Kochel, M.M. Plichta, A. Schafer, V. Leutgeb, W. Scharmuller, A.J. Fallgatter, and A. Schienle, "Affective perception and imagery: A NIRS study," International Journal of Psychophysiology, vol. 80, no. 3, pp. 192-197, June 2011.

[10] S. Sasai, F. Homae, H. Watanabe, and G. Taga, "Frequency-specific functional connectivity in the brain during resting state revealed by NIRS," NeuroImage, vol. 56, no. 1, pp. 252-275, 2011.

[11] Leonard Smith, Chaos: A very short introduction, Oxford University Press, 2007.

[12] H.A. Sturges, "The choice of a class interval," J. American Statistical Association, vol. 21, no. 153, pp. 65-66, March 1926.

[13] D.W. Scott, "On optimal and data-based histograms," Biometrika, vol. 66, no. 3, pp. 605-610, 1979.

[14] D. Freedman and P. Diaconis, "On the histogram as a density estimator: $L_{2}$ Theory," Zeitschrift fur Wahrscheinlichkeitstheorie und Verwandte Gebiete, vol. 57, no. 4, pp. 453-476, December 1981.

[15] "Detecting and evaulating intrinsic nonlinearlity present in the mutual dependence between two variables," Physica D, vol. 147, pp. 1-11, 2000.

[16] K. Aihara, J. Imura, and T. Ueta, Analysis and control of complex dynamical systems, Springer, 2015.

[17] F. Takens, "Detecting strange attractors in turbulence" in Dynamical systems and Turbulence, ed. D.A. Rand and L.S. Young, pp. 366-381, Springer, Berlin, 1981.

[18] T. Sauser, J.A. Yorke, and M. Casdagli, "Embedology," J. Stat. Phys., vol. 65, no. 3-4, pp. 579616, 1991. 
[19] M.B. Kennel and S. Isabelle, "Methods to distinguish possible chaos from colored noise and to determine embedding parameters," Physical Review A, vol. 45, no. 6, pp. 3403-3411, March 1992.

[20] M.B. Kennel, R. Brown, and H.D.I. Abarbanel, "Determining embedding dimension for phasespace reconstruction using a geometrical consruction," Physical Review A, vol. 45, no. 6, pp. 3403-3411, March 1992.

[21] H.D.I. Abarbanel and M.B. Kennel, "Loca false nearest neighbors and dynamical dimensions from observed chaotic data," Physical Review E, vol. 47, no. 5, pp. 3057-3068, May 1993.

[22] H.D.I. Abarbanel, Analysis of Observed Chaotic Data, Springer, October 1995.

[23] P. Eckmann, S. Kamphorst, and D. Ruelle, "Recurrence plots of dynamical systems," Europhys. Letters, vol. 5, pp. 973-977, 1987.

[24] N. Marwan, M. Romano, M. Thiel, and J. Kurths, "Recurrence plots for the analysis of complex systems," Physics Reports, vol. 438, no. 5, pp. 237-329, 2007.

[25] L. Charles, Jr. Webber, and N. Marwan, Recurrence Quantification Analysis: Theory and Best Practices (Understanding Complex Systems), Springer-Verlag, 2014.

[26] N. Marwan, "How to avoid potential pitfalls in recurrence plot based data analysis," Int. J of Bifurcation and Chaos, vol. 21, no. 4, 2011.

[27] K. Iwayama, Y. Hirata, K. Takahashi, K. Watanabe, K. Aihara, and H. Suzuki, "Characterizing global evolutions of complex systems via intermediate network representations," Scientific Reports, vol. 2, 423, 2012.

[28] J. Iwanski and E. Bradley, "Recurrence plots of experimental data: To embed or not to embed?," Chaos, vol. 8, pp. 861-871, 1998.

[29] M. Bianciardi, P. Sirabella, G.E. Hagberg, A. Giuliani, J.P. Zbilut, and A. Colosimo, "Modelfree analysis of brain fMRI data by recurrence quantification," NeuroImage, vol. 37, no. 2, pp. 489-503, August 2007.

[30] T. Miao, T. Hashiguchi, and I. Maruyama, "Nonlinear analysis of heart rate variability and plethysmogram in subjects with normal and abnormal cardiovascular function," Proc. SMC '06, pp. 2885-2890, 2006.

[31] D.A. Rosenbaum, Human Motor Control, Academic Press, 2010.

[32] R.W. Homan, J. Hermanb, and P. Purdy, "Cerebral location of international 10-20 system electrode placement," Electroencephalography and Clinical Neurophysiology, vol. 66, no. 4, 1987.

[33] J. Gibbons, Nonparametric Statistics (Quantitative Applications in the Social Sciences), SAGE University Press, 1992.

[34] H. Scheffe, The analysis of variance, Wiley, 1999.

[35] T.Q. Khoa, Y. Nakamura, and M. Nakagawa, "Recognizing brain motor imagery activities by identifying chaos properties of oxy-hemoglobin dnamics tie series," Chaos, Solitons 83 Fractals, vol. 42, no. 1, 2009.

[36] X. Hu, K. Hong, and S. Ge, "Recognition of stimulus-evoked neuronal optical response by identifying chaos levels of near-infrared spectroscopy time series," Neuroscience letters, vol. 504, no. 2, pp. 115-120, October 2011.

[37] T. Funane, H. Atsumori, T. Katura, A. Obata, H. Sato, Y. Tanikawa, E. Okada, and M. Kiguchi, "Quantitive evaluation of deep and shallow tissue layers' contribution to fNIRS signal using multi-distance optodes and independent component analysis," NeuroImage, vol. 85, no. 1, pp. 150-165, 2014.

[38] T. Fekete, D. Ubin, J.M. Carlson, and L.R. Mujica-Parodi, "The NIRS analysis package: noise reduction and statistical inference," PLOS ONE, vol. 6, no. 9, e24322, September 2011.

[39] J. Ashe, O.V. Lungu, A.T. Basford, and X. Lu, "Cortical control of motor sequence," Current Opinion of Neurobiology, vol. 16, no. 2, pp. 213-221, April 2006.

[40] C. Bogler, J. Mehnert, J. Steinbrink, and J. Haynes, "Decoding vigilance with NIRS," PLoS ONE, vol. 9, pp. 1-12, 2014. 